\title{
RESSECÇÃO DE MELANOMA LENTIGINOSO ACRAL COM RECONSTRUÇÃO DE RETALHO SURAL DE FLUXO REVERSO - RELATO DE CASO
}

\author{
RESECTION OF ACRAL LENTIGINOUS MELANOMA WITH REVERSER FLOW RECTAL \\ RECONSTRUCTION - CASE REPORT
}

\author{
Paulo Henrique Ribeiro de Oliveira', Livia Nunes Barboza Duarte² ${ }^{2}$ Barbara Paula dos Santos Batista ${ }^{3}$, Haroldo \\ José Siqueira da Igreja Junior ${ }^{4}$ \\ 1 Residente serviço de Cancerologia Cirúrgica do Hospital Escola Álvaro Alvim, Campos - RJ. \\ 2 Acadêmica de medicina da Faculdade de Medicina de Campos - RJ \\ 3Pós-doutoranda em Medicina Veterinária (Clínica e Reprodução animal), pela Universidade Federal \\ Fluminense, Niterói -RJ \\ 4Supervisor do Programa de Residência Médica de Cancerologia Cirúrgica, do Hospital Escola Álvaro Alvim, \\ Campos dos Goytacazes - RJ
}

Trabalho realizado no Serviço de Cancerologia Cirúrgica do Hospital Escola Álvaro Alvim, situado na Rua Barão da Lagoa Dourada, 409, Centro, Campos dos Goytacazes RJ. CEP: 28035-211. Telefone: (22) 2726-6700 Endereço de correspondência: Rua Barão da Lagoa Dourada, 409, Centro, Campos dos Goytacazes - RJ. CEP: 28035-211. e-mail: dr.paulohenriqueoliveira@gmail.com

\section{RESUMO}

O melanoma maligno é uma neoplasia cutânea com elevada agressividade e mortalidade que pode afetar todas as idades. Os fatores de risco conhecidos são ambientais, individuais e genéticos. O melanoma lentiginoso acral é um subtipo raro de melanoma e atinge de forma desproporcional pessoas com classificação Fitzpatrick diferente. 0 melanoma lentiginoso acral tem um pior prognóstico comparado aos outros subtipos, e isso foi atribuído ao seu comportamento biológico agressivo. A excisão cirúrgica com margem adequada é a única abordagem com potencial curativo em casos de melanoma maligno. Este artigo descreve um relato de caso sobre a técnica de ressecção do melanoma lentiginoso acral em associação a reconstrução do retalho sural de fluxo reverso, com intuito de ressaltar a importância desta opção de tratamento cirúrgico em uma área anatomicamente de difícil reconstrução cirúrgica. As informações foram obtidas dos prontuários do Hospital Escola Álvaro Alvim, Campos dos Goytacazes, Rio de Janeiro, no

\begin{abstract}
Malignant melanoma is a skin cancer with high aggressiveness and mortality that can affect all ages. The known risk factors are environmental, individual and genetic. Acral lentiginous melanoma is a rare subtype of melanoma and disproportionately affects people with a different Fitzpatrick classification. Acral lentiginous melanoma has a worse prognosis compared to other subtypes, and this was attributed to its aggressive biological behavior. Surgical excision with an adequate margin is the only approach with curative potential in cases of malignant melanoma. This article describes a case report on the technique of resection of acral lentiginous melanoma in association with reconstruction of the reverse flow sural flap, in order to highlight the importance of this surgical treatment option in an anatomically difficult area of surgical reconstruction. Information was obtained from the medical records of Hospital Escola Álvaro Alvim, Campos dos Goytacazes, Rio de Janeiro, from 12/01/2017 to 01/31/2021. A 44-yearold female patient, with Fitzpatrick
\end{abstract}




\section{INTRODUÇÃO}

O melanoma é decorrente de mutações genéticas nos melanócitos, que são as células produtoras de pigmento, que podem ser encontradas na pele, olhos, ouvido interno e leptomeninges. A incidência do melanoma continua aumentando, principalmente em indivíduos com mais de 65 anos. Existem quatro tipos principais de melanoma, que correspondem a quase $100 \%$ dos casos diagnosticados: Melanoma de disseminação superficial, Melanoma nodular, Melanoma lentigo maligno e Melanoma lentiginoso acral .

O melanoma lentiginoso acral, o menos comum dos quatros principais subtipos de melanoma maligno cutâneo, foi descrito pela primeira vez por Reed em 1976. E como o nome sugere o melanoma acral ocorre na pele acral, ou seja, nas palmas, plantas e leito ungueal. É um tumor cutâneo incomum que ocorre em todas as raças na mesma frequência.

A cobertura cutânea do terço distal da perna, tornozelo e pé, permanece um desafio para cirurgia reconstrutiva . Essas áreas são suscetíveis à traumas e possuem particularidades anatômicas, como a falta de um envelope muscular sobre os ossos, aumentando o risco de problemas quanto a sua cobertura, juntamente com suas novas estruturas. Neste ponto o retalho fasciomiocutâneo sural de fluxo reverso, se apresenta como um importante aliado do cirurgião oncológico no tratamento do melanoma acral.

\section{OBJETIVOS}

Descrever de um relato de caso sobre a técnica de ressecção do melanoma lentiginoso acral em associação a reconstrução do retalho sural de fluxo reverso, com intuito de ressaltar a importância desta opção de tratamento cirúrgico em uma área anatomicamente de difícil reconstrução cirúrgica.

\section{DESCRIÇÃO DOCASO}

Paciente do sexo feminino, 44 anos, de fototipo $V$ de Fitzpatrick, sem antecedentes pessoais ou familiares de melanoma. Foi encaminhada ao serviço de oncologia clínica do Hospital Escola Álvaro Alvim, com diagnóstico prévio de melanoma in situ de padrão lentiginoso, por meio de biópsia incisional, realizado fora do Hospital Escola Álvaro Alvim.
Após primeira consulta foi direcionada para o serviço de cirurgia oncológica.

Os diâmetros da lesão, correspondiam aproximadamente $45 \times 35 \mathrm{~mm}$, localizada em região calcânea esquerda, com evolução relatada em um período de dois anos e crescimento rápido nos últimos dois meses. A região apresentava-se enegrecida, assimétrica e com bordas irregulares (Fig. 1).

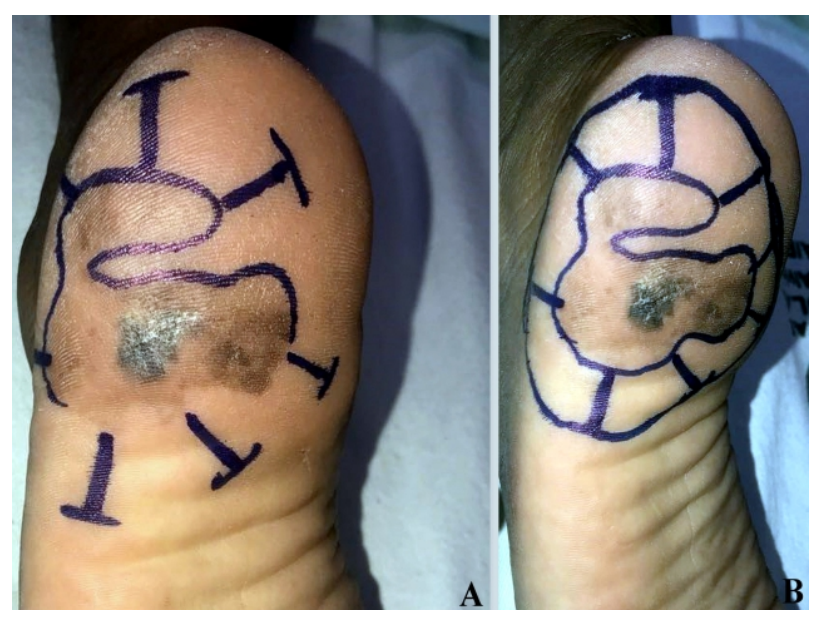

Figura 1: Melanoma in situ

O exame físico não foi observado linfonodomegalia, e no estadiamento clínico préoperatório da paciente, que incluíram a tomografia de tórax, abdômen e pelve, não foi evidenciado doença à distância.

A cirurgia iniciou com a demarcação da lesão melanocítica foi por meio de uma caneta própria para o centro cirúrgico, realizando uma margem de $20 \mathrm{~mm}$ a partir de toda a borda da lesão (Fig. 2A).

A incisão foi realizada com bisturi na pele até subcutâneo da área demarcada, em seguida foi ressecado em bloco, pele, subcutânea e fáscia plantar. O material ressecado foi enviado para avaliação histopatológica (Fig. 2B, 2C).

$O$ procedimento de reconstrução iniciou com realização da medição do tamanho da região ressecada e criação de um gabarito do retalho da região doadora. Para determinar o comprimento do pedículo, foi definido o ponto de rotação do pedículo vascular (5 $\mathrm{cm}$ acima do maléolo lateral), a localização da última artéria perfurante mais calibrosa (artéria fibular), e a área que irá receber o retalho sural de fluxo reverso. 


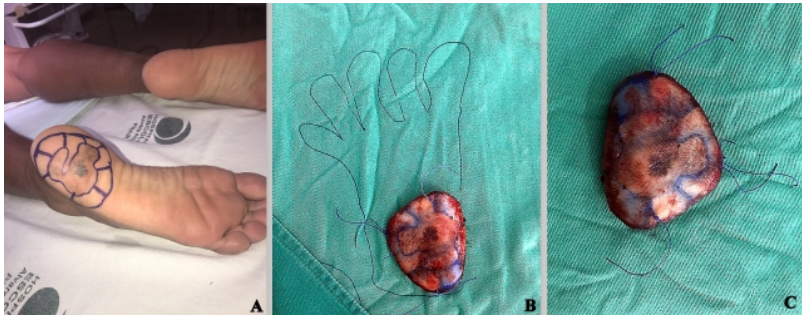

Figura 2 : Ressecção do melanoma

Com auxílio de uma caneta demarcadora de uso cirúrgico, foram transferidas as medidas para o eixo central da região posterior da perna esquerda. Foi realizada a incisão na borda superior do retalho, entre a porção medial e lateral dos músculos gastrocnêmios.

A dissecção do subcutâneo foi realizada com auxílio de uma tesoura de metzembaum, em sentido biselada e ascendente, para confecção da borda fasciossubcutânea maior da pele do retalho.

A veia safena parva e o nervo cutâneo medial da sura foram identificados, dissecados, ligados e seccionados.

Iniciou-se à dissecção do retalho, da região proximal para distal, sempre levando em bloco, fáscia profunda, nervo medial da sura e veia safena parva.

Após dissecção de toda extensão do retalho, continuou com dissecção do pedículo até ponto acima do maléolo lateral, nesse ponto o pedículo sofrerá rotação de 180 이․ $3 \mathrm{~A}$ ). A dissecção da pele e do subcutâneo continuou até o ponto de implantação do retalho, criando um túnel que abrigou o pedículo vascular.

O retalho sural alcançou seu ponto de implantação sem tensão superficial, sendo fixado em seu leito com pontos de fios de vicril 2.0 (Fig. 3B).

$O$ trajeto da incisão da pele para a dissecção do pedículo vascular foi suturado com fio de nylon 3.0 (Fig. 3C). A área doadora do retalho sural necessitou de um enxerto de pele (região infraglútea) para o seu fechamento.

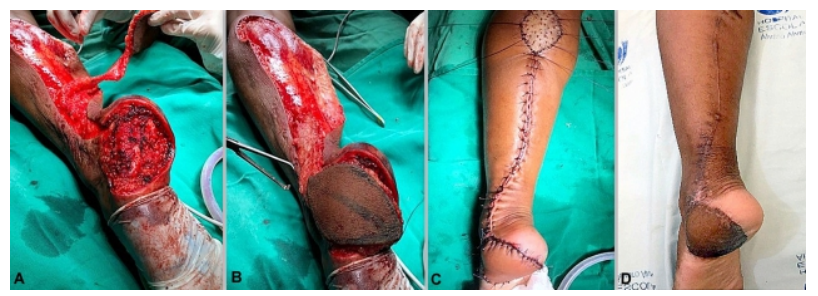

Figura 3: Retalho sural de fluxo reverso

O histopatológico do material resseccionado, confirmou o diagnóstico de melanoma in situ de padrão lentiginoso acral, em fase de crescimento radial, nível de infiltração Clark I, atividade mitótica não detectada, infiltração linfocitária peritumoral escassa, áreas de regressão ausente, satelitose microscópica e ulceração não detectadas.

\section{CONCLUSÃO}

No caso descrito, em que a superfície plantar sustenta o peso corporal, o enxerto de pele por vezes não apresenta boa cicatrização. $O$ retalho sural de fluxo reverso foi utilizado para a cobertura da grande falha tecidual provocada pela ressecção do melanoma obedecendo a critérios de tratamento neoplásicos. O tratamento cirúrgico do melanoma acral representa um desafio para o cirurgião, visando à ressecção total da lesão, com objetivo de curar e prevenir as recidivas.

\section{REFERÊNCIAS}

1. Tolleson, W.H. Human melanocyte biology, toxicology, and pathology. J. Environ. Sci. Health C Environ. Carcinog. Ecotoxicol. Rev. 23, 105-161 (2005).

2. Gray-Schopfer, V., Wellbrock, C. \& Marais, R. Melanoma biology and new targeted therapy. Nature 445, 851-857 (2007).

3. Populo, H., Soares, P. \& Lopes, J.M. Insights into melanoma: targeting the mTOR pathway for therapeutics. Expert opinion on therapeutic targets 16, 689-705 (2012).

4. L, D. \& B, K. The menace of melanoma: a photodynamic approach to adjunctive cancer therapy. In:

G, H.T.D. (ed). Melanoma - From Early Detection to Treatment. INTECH Open Access Croacia, 2013.

5. Holland, J.F. \& Pollock, R.E. Holland-Frei cancer medicine 8 vol. 8. PMPH-USA, 2010.

6. Reed, R.J. New Concepts in Surgical Pathology of the Skin. . John Wiley: New York, 1976. 
7. Bristow, I.R. \& Acland, K. Acral lentiginous melanoma of the foot and ankle: A case series and review of the literature. J Foot Ankle Res 1, 11 (2008).

8. Hao, X. et al. Acral Lentiginous Melanoma of Foot and Ankle: A Clinicopathological Study of 7 Cases. Anticancer research 39, 6175-6181 (2019).

9. Li, B., Chang, S.M., Du, S.C., Zhuang, L. \& Hu, S.J. Distally Based Sural Adipofascial Turnover Flap for Coverage of Complicated Wound in the Foot and Ankle Region. Annals of plastic surgery 84, 580-587 (2020).

10. Rajacic, N., Darweesh, M., Jayakrishnan, K., Gang, R.K. \& Jojic, S. The distally based superficial sural flap for reconstruction of the lower leg and foot. British journal of plastic surgery 49, 383-389 (1996).

11. Ajmal, S. et al. Distally based sural fasciocutaneous flap for soft tissue reconstruction of the distal leg, ankle and foot defects. J Ayub Med Coll Abbottabad 21, 19-23 (2009).

12. Rezende, M.R. et al. Skin coverage of the middle-distal segment of the leg with a pedicled perforator flap. Journal of orthopaedic trauma 24, 236-243 (2010). 